\title{
Wissen mal ganz konventionell unkonventionell
}

Stadt verstehen mit Lucius Burckhardt

Reto Bürgin

Abb. 1 Lucius Burckhardt (Foto: Annemarie Burckhardt. (C) Martin Schmitz Verlag[1])

„Vor Ort sieht die Welt ganz anders aus als am Schreibtisch.“ (Burckhardt 1994: 6)

„Können wir die Stadt wahrnehmen?“ fragte sich Lucius Burckhardt (1994; 2017). Der Schweizer Soziologe und kritische Grenzgänger (vgl. Gribat 2017) beschäftigte sich intensiv mit dieser Frage. Als Professor für ,Sozioökonomie urbaner Systeme“ in Kassel gründete er zusammen mit seiner Frau Annemarie Burckhardt-Wackernagel die sogenannte, Spaziergangswissenschaft" - auch ,Promenadologie' genannt (vgl. Burckhardt 1995).

Spaziergänge dienen dabei als didaktisches Mittel zur Schärfung der Wahrnehmung von Landschaft (Schoch/Bossert 2017). Aber wie? In zahlreichen Spaziergängen versuchte Burckhardt, den kritischen Blick seiner Studierenden zu schärfen und ihre konventionelle Wahrnehmung von Landschaft zu hinterfragen. Dabei war es nicht von Belang, ob die Landschaft ländlich oder städtisch geprägt war.

Dieser Beitrag rückt Burckhardts Spaziergangswissenschaft in den Mittelpunkt des Interesses. Dabei diskutiere ich deren methodologischen Gewinn

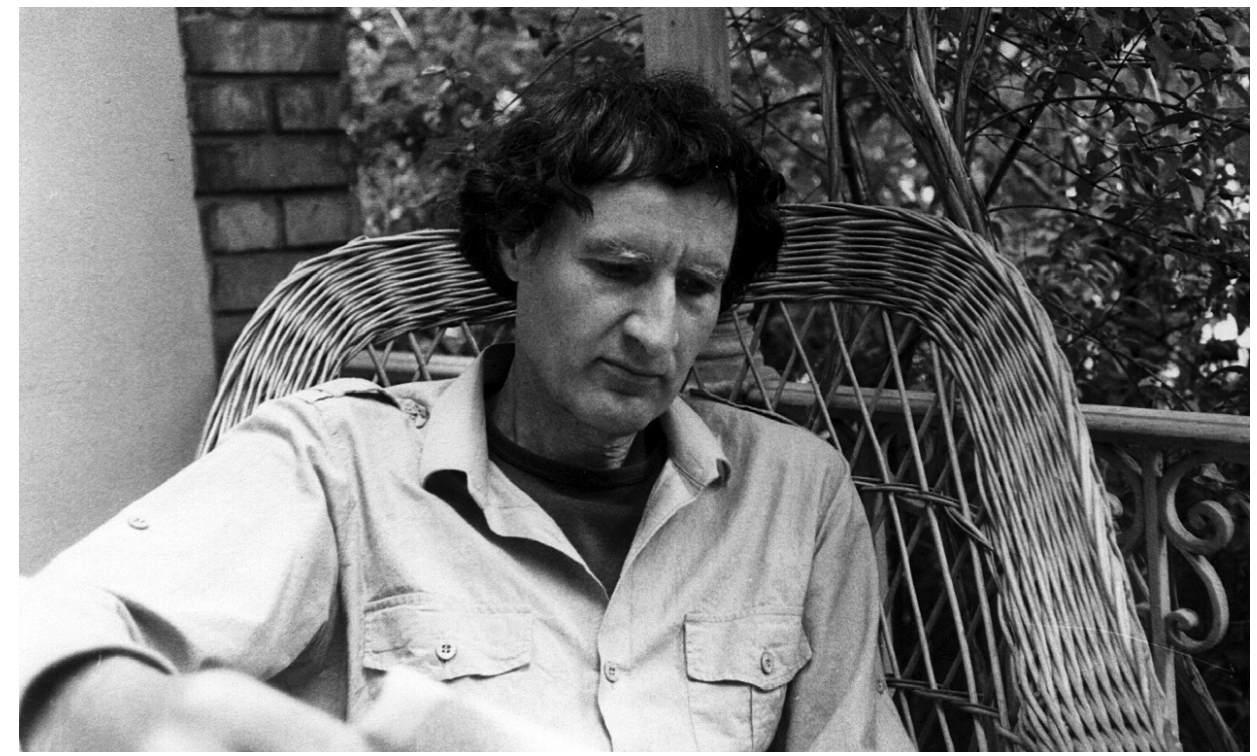


für die Erforschung und Generierung neuen räumlichen Wissens. Zunächst gehe ich auf Burckhardts systemische Denkweise ein. In einem weiteren Schritt nähere ich mich Burckhardts Spaziergang an. Darauffolgend reflektiere ich die Spaziergangswissenschaft als mögliche Methode für die Wissensgenerierung.

\section{Eine unkonventionelle Denkweise}

In seinem Aufsatz „Wer plant die Planung?“ (1974)[2] unternahm Burckhardt den Versuch, den planerischen Prozess in seine Einzelteile zu zerlegen. Er hinterfragte das Machtverhältnis zwischen unterschiedlichen Akteur_innen in Planungsvorhaben. In diesem ,Kräfteparallelogramm“ versuchte Burckhardt, Ordnung beziehungsweise Systematik in den Nebel der Entscheidungsfindung zu bringen. Der Blick schweifte somit vom Fokus auf die Ausgangslage und das planerische Endprodukt auf die dahinterliegenden Beziehungen, Mechanismen und Prozesse. Planung ist dabei „nicht autonom, sondern einem sozialen System zugehörig und wirksam durch ein Personenkollektiv, eine Organisation“ (Burckhardt 1974: 87). Somit ist Planung als ein mehrteiliges System zu verstehen, das nicht nur die finale architektonische Formgebung, sondern auch soziale Komponenten miteinbezieht.

Mit seiner systemischen Sichtweise auf Planung kritisierte Burckhardt den Planungsprozess. So komme es immer wieder vor, dass Architekt_innen, die in Burckhardts Verständnis bis in die späten sechziger Jahre auch Planer_innen waren, intuitive Entscheidungen treffen, wobei die Komplexität einer Aufgabe beziehungsweise eines Problems stets auf das ,Wesentliche reduziert würde. Nach Burckhardt führt dies zu einer problematischen und trügerischen „Reduktion komplizierter Problemzusammenhänge“ (Burckhardt 1974: 75). Damit ist gemeint, dass der Umgang mit dem planerischen Kontext eines Problemkomplexes zunächst nicht zum Handwerk von Architekt_innen gehörte und somit für sie als, unwesentlich' gelten würde. In der Folge fällt dieser planerische Kontext „unter den Tisch“ (ebd.) und erhält somit kaum deren Beachtung. Doch gerade dieser unter den Tisch gefallene Kontext sollte nicht in Vergessenheit geraten und spielte für Burckhardt eine wichtige Rolle.

Schon sieben Jahre zuvor gliederte Burckhardt (1967) in seinem Aufsatz „Bauen - ein Prozess ohne Denkmalpflichten“ systematisch den Bau- und Planungsprozess in ein relationales Dreieck mit den Eckpunkten Mensch, Umwelt und Politik. Mit dieser „kleinen Dreitaktmaschine“ (ebd.: 32) erklärte Burckhardt anhand der sogenannten „Spiegelsituation“(ebd.: 29), wie der Mensch die Umwelt verändert und vice versa: Die drei Eckpunkte können dabei nicht isoliert voneinander betrachtet werden, denn das Ganze ist in der Realität „ein in sich verflochtenes System“ (ebd.: 29). Mit der Dreieckskonstellation verdeutlichte Burckhardt, dass der Weg von Veränderungen, ganz gleichgültig ob von Mensch oder Umwelt ausgehend, stets über den Eckpunkt Politik abläuft. Burckhardt erklärte somit anhand des Dreieckmodells, wie planerische und bauliche Veränderungen vonstattengehen. Dabei ist es wichtig, alle Eckpunkte gleich ernst zu nehmen. Burckhardt fokussierte sich also nicht gänzlich auf das Objekt beziehungsweise die Lösung des 
vordergründigen Problems, sondern vielmehr auf dahinterliegende soziale und politische Prozesse, die zu Veränderung führen.

Lucius Burckhardt richtete seinen Blick stets hinter das visuell Sichtbare und versuchte dabei Sachverhalte systematisch in Einzelteile zu gliedern (vgl. Bürgin 2019). Dies zeigt sich in seinen Aufsätzen zum unsichtbaren Design. Der Aufsatz „Design ist unsichtbar“ (Burckhardt 1980) war eine Weiterführung seiner Planungskritik. Darin kritisierte er das dem damaligen deutschen Werkbund zugrundeliegende Paradigma der, Guten Form': Design solle nicht nur als Form eines Objekts verstanden werden, sondern auch deren soziale Komponenten miteinbeziehen und ernst nehmen. Denn gerade der soziale Aspekt von Design scheine auf den ersten Blick unsichtbar zu sein. Mit dieser Sichtweise animierte Burckhardt dazu, den Blick nicht nur auf die Dinge selbst zu richten, sondern auch auf die dahinterliegenden unsichtbaren Regelungen und organisatorischen Beziehungen. Dabei ist die Umwelt als ein System zu verstehen, das aus Subsystemen besteht. Der Fokus verlagert sich somit weg vom Einzelobjekt und hebt dessen soziale Wirkung hervor (vgl. Bürgin 2019). Diese hier nur knapp angedeutete systemische Denkweise zieht sich durch Burckhardts gesamtes hinterlassenes Werk und ist auch in seiner eigens begründeten Spaziergangswissenschaft wiederzuerkennen.

\section{Zu Fuß die Welt erkunden - und verstehen}

Eine der bekanntesten Hinterlassenschaften von Lucius Burckhardt ist die sogenannte, Spaziergangswissenschaft'. Der Spaziergang, die Praxis der langsamen Fortbewegung zu Fuß, fungiert dabei ,als Urform der Wahrnehmung in Bewegung " (Ritter 2019: 22). Durch die langsame Fortbewegung zu Fuß ist die sinnliche und visuelle Wahrnehmung der Umwelt stärker ausgeprägt. Der temporale Aspekt des ,Langsamseins ' ist dabei zentral, wodurch der Spaziergang auch als ,anachronistische Geste der Entschleunigung“ zu verstehen ist (Schoch/Bossert 2017: 322). Wer mit dem Auto oder mit dem Zug fährt, hat kaum die Möglichkeit, die gesehenen Eindrücke im Kopf zu verarbeiten: „Moderne Verkehrsmittel neigen dazu, die Reise unbedeutend und abstrakt werden zu lassen und allein das Ziel in den Vordergrund zu stellen." (Burckhardt 1995: 266) Dabei geht das Erlebnis des Weges verloren. Eine kritische, tiefgründige Aufnahme und Reflexion der visuellen Betrachtung scheint somit zur Herausforderung zu werden. Die Kritik gilt hier vermutlich vorweg nicht in der Wahl der Verkehrsmittel, sondern eher der unreflektierten Abstraktion der Reise und der Ausrichtung auf das Erlebnis am Zielort. So galt es für Burckhardt gerade auch, diese Abstraktheit systematisch zu zerlegen und die dahinterliegenden Prozesse und Dynamiken zu erkunden. Der Spaziergang ist jedoch in Burckhardts Verständnis nicht als Darstellung, sondern als eine Wahrnehmung zu verstehen (Burckhardt 1995: 257). Sie soll anregen, den Ist-Zustand zu begreifen und zu hinterfragen sowie auch zum Träumen anzustiften (Bürgin/Schoch 2015: 78).

Burckhardt war aber nicht der Erste, der sich mit der „Bewegung als Praxis“ für die Gewinnung neuer Erkenntnisse auseinandersetzte (Bossert 2014: 142). Schon vor seiner Zeit wurde die Mobilität zu Fuß für die Erlebnis- und Wissensgenerierung eingesetzt. Davon zeugen Pilgerfahrten von Gläubigen an heilige Orte oder Reisen, wie zum Beispiel die Odyssee. 
Auch Sokrates ' und Rousseaus Spaziergänge dienen als Beispiele dafür. In jüngerer Zeit war es der Soziologe Robert E. Park, der unter anderem seine Studierenden dazu animierte, zu Fuß Chicago zu erkunden und zu beobachten. Auch Walter Benjamin mit der Figur des Flaneurs und die Situationistische Internationale mit der Psychogeographie reihen sich in die Gruppe der wissenssuchenden Spazierenden ein (dazu ausführlicher: Bossert 2014; Bürgin/Schoch 2015; Schoch/Bossert 2017). Spuren dieser Vorläufer sind auch in Burckhardts Land- und Stadtspaziergängen wiederzufinden. Aber was ist bei Burckhardt neu? „Es ist die einmalige Kombination all dieser Elemente. Lucius Burckhardts Originalität liegt im Versuch, Kunst, Kulturtechnik und wissenschaftliche Methode zu vereinen. Die Spazierenden werden sinnlich, habituell und intellektuell angesprochen. Lucius Burckhardt hofft so, bis zu seinen Mitmenschen vorzudringen, damit neue Erkenntnisse und eine veränderte Wahrnehmung haften bleiben.“ (Bossert 2014: 145)

Anstatt jedoch zu explizit auf das Vergangene aufzubauen, gründete Burckhardt kurzum seine eigene Wissenschaft des Spazierens, die auch als Methode eines Aktionsunterrichts zu verstehen ist. Mit Performances, Exkursionen und spontanen Aktivitäten versuchte er dabei, den kritischen Blick seiner Mitmenschen zu schärfen. Mit einer Prise Humor und einem feinen Gespür dafür, andere zu begeistern, unternahm er zahlreiche Spaziergänge, führte ein Seminar auf einem Parkplatz durch, organisierte künstlerische Performances in der Schweizer Bergwelt, regte mit Wortspielen zum Nachdenken an, kennzeichnete mit einem Schild den Anfang der Landschaft oder rollte einen überdimensionalen Zebrastreifen in der Kasseler Innenstadt aus. Solche Aktionen, die auch einen gewissen Eventcharakter hatten, lassen sich als Methoden für einen bewusst inszenierten Perspektivenwechsel und zur Schärfung des Blicks verstehen. Aber auch die zu den Performances dazugehörenden Begriffe und Wortspiele wie ,Denkmal Nach', die ,Landschaftsfalle“ oder das ,ZEBRA streifen“ sollten zum Nachdenken anregen.
Abb. 2 Das ZEBRA streifen, Kassel 1993 (Foto: Angela Siever. (C) Martin Schmitz Verlag)

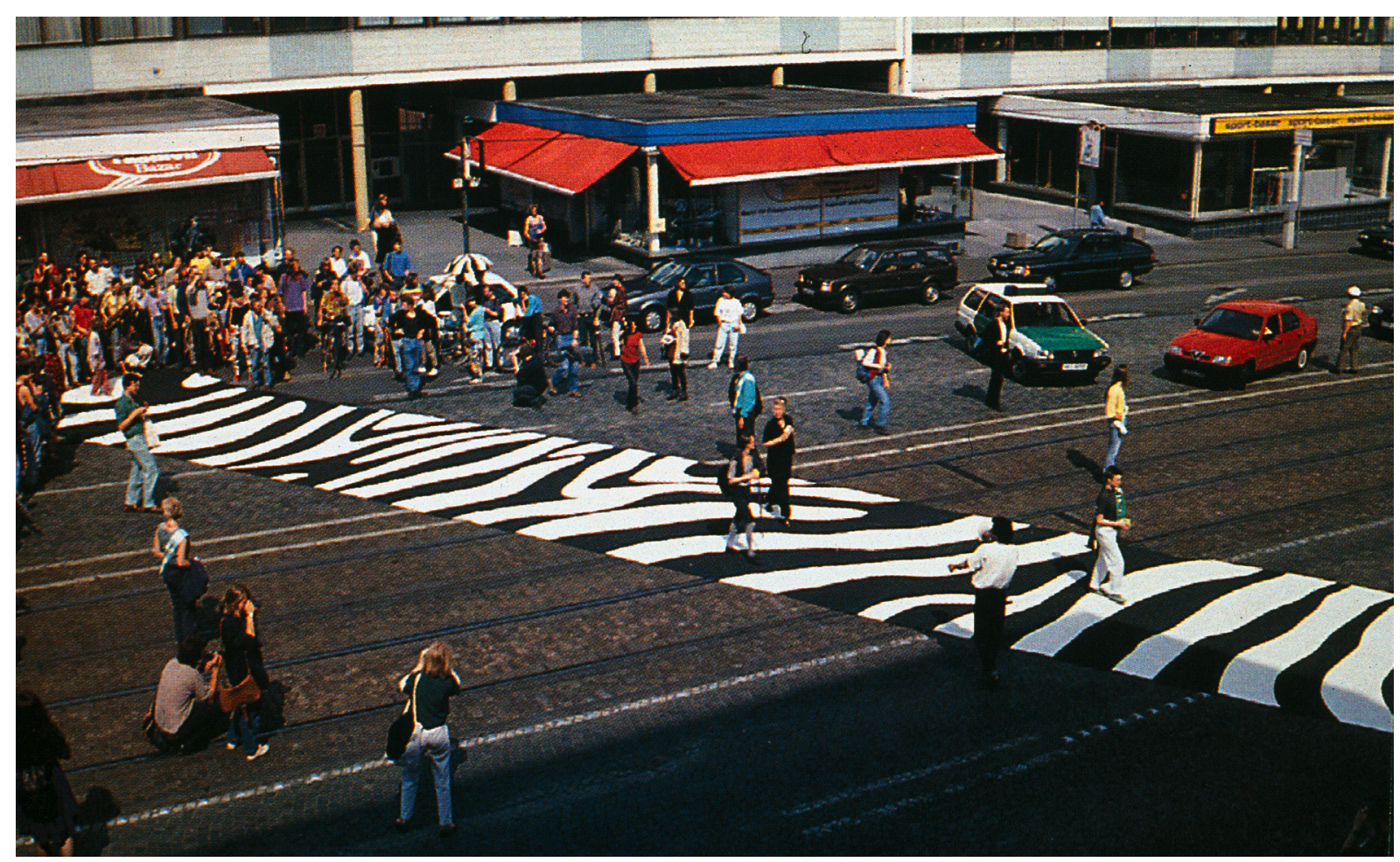




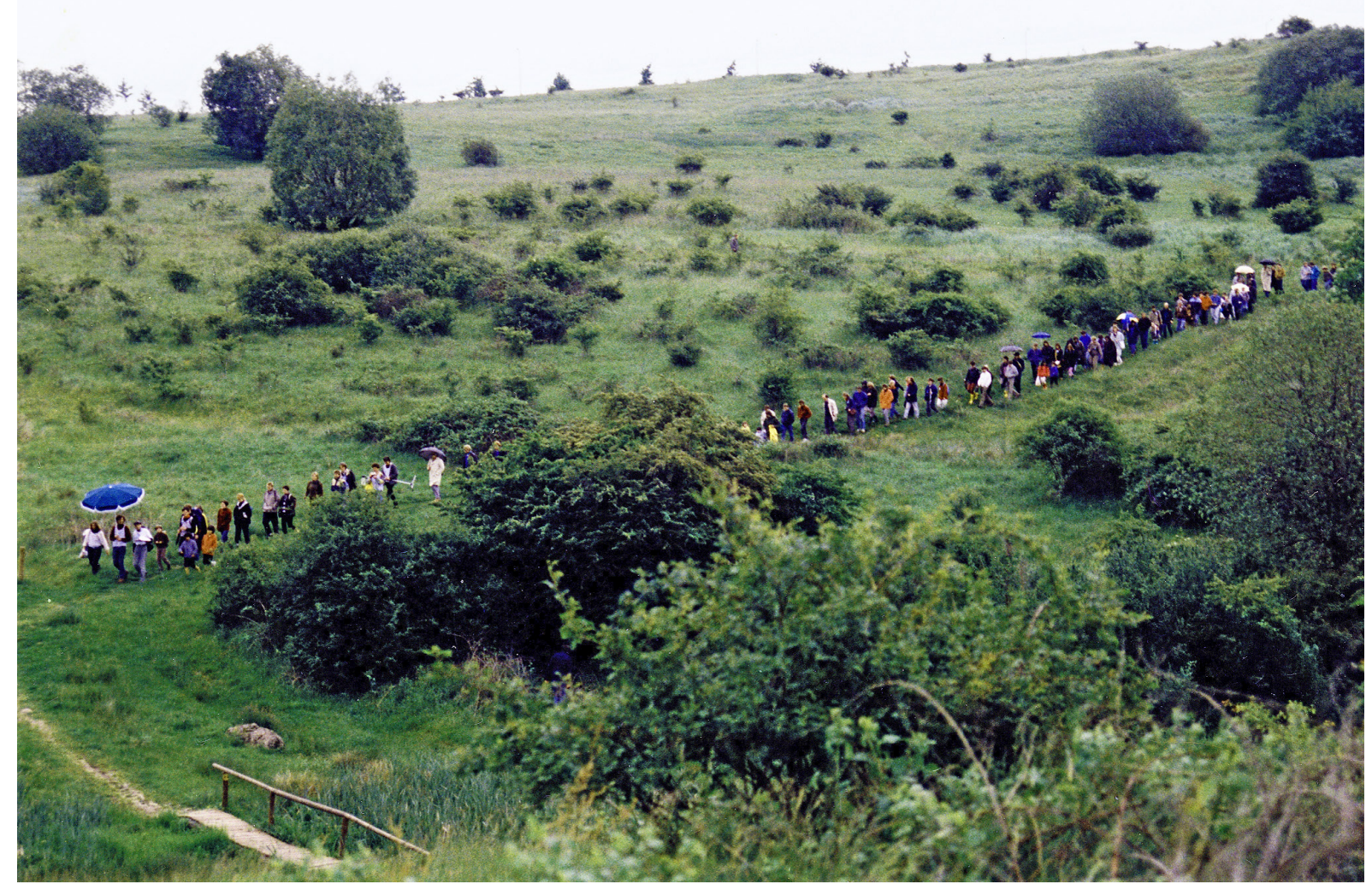

Abb. 3 Die Fahrt nach Tahiti, Kassel 1987 (Foto: Klaus Hoppe. (C) Martin Schmitz Verlag)
Einer von Burckhardts bekanntesten Spaziergängen ist die ,Fahrt nach Tahiti', die er 1987 anstatt auf die Insel Tahiti im Naturschutzgebiet der Kasseler Dönche durchführte. Der Spaziergang war als Kritik am konservativen Naturschutz gedacht (Bossert 2014: 139). Der ehemalige Truppenübungsplatz der Bundeswehr fungierte dafür als Kulisse, obwohl er auf den ersten Blick in keiner Weise der paradiesischen Insel im Südpazifik zu gleichen schien. Im Laufe des Spaziergangs wurden Auszüge aus dem Reisebericht des Naturforschers und Ethnologen Georg Forster vorgelesen, der im Jahr 1772 mit Captain Cook nach Tahiti gesegelt war. Burckhardt verblüffte seine Mitspazierenden. Denn der von Forster beschriebene Brotbaum war auch in der Dönche anzutreffen. Der Baum sah einfach ein bisschen anders aus: Die Brote stammten aus einer Bäckerei und waren daran aufgehängt. Für die Spazierenden war es erstaunlich, wie die Beschreibungen Forsters auch auf die Kasseler Dönche zutrafen. Während der performative Aspekt das Rahmenprogramm vorgab, war die eigentliche Intention des Spaziergangs eine andere. Burckhardt versuchte anhand des Spaziergangs in Kombination mit Forsters Lektüre zu zeigen, wie Landschaft wahrgenommen wird und dass Umgebungen mental konstruierte Kreationen in unseren Köpfen sind (vgl. Burckhardt 1995; Bossert 2014; Bürgin/Schoch 2015).

Ein nicht minder bekannter Ausflug zu Fuß war der Autofahrerspaziergang. Die Studierenden spazierten mit einer vor die Gesichter gehaltenen Autowindschutzscheibe durch die Straßen von Kassel. Sinn und Zweck dieser Aktion war es, sich der eingeschränkten Perspektive von Autofahrenden bewusst zu werden und Raum ohne Auto erfahrbar zu machen (vgl. Sutter 2014: 50 ff.).

Bei seinen Events konnte Burckhardt experimentieren und Neues ergründen, indem er versuchte, das real und physisch Gesehene als etwas 


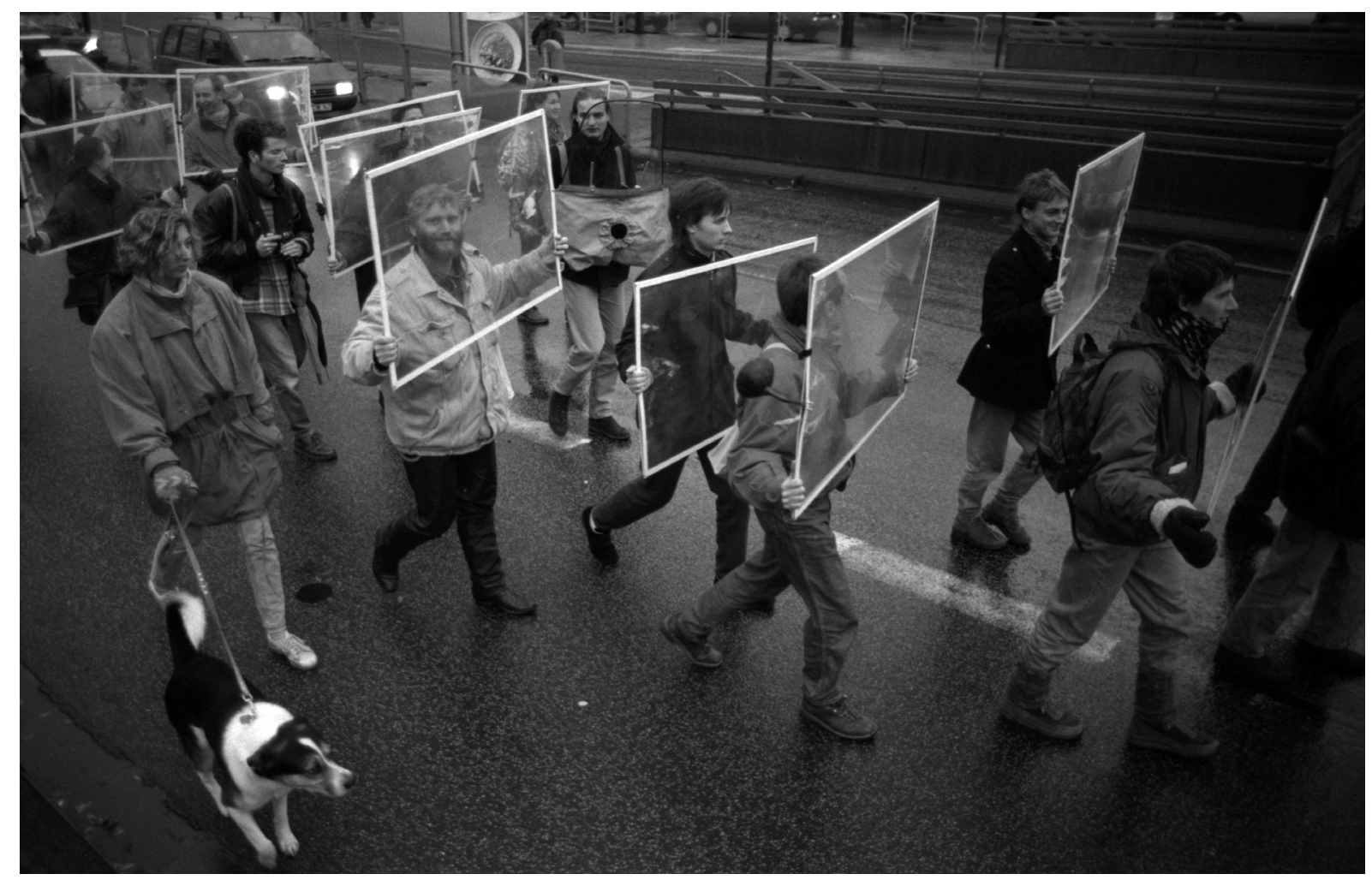

Konstruiertes darzustellen. Mit solchen, mal mehr und mal weniger aufwändigen performativen Aktionen induzierte Burckhardt bewusst einen Überraschungs- und Lerneffekt bei den Spazierenden. Somit fungierte die Spaziergangswissenschaft als eine Art Schulung der Wahrnehmung, welche aber dann insbesondere in den Diskussionen zwischen den Spazierenden erweitert und geschärft wurde.

\section{Spazieren als wissenschaftliche Methode?}

Burckhardts Spaziergänge sind in Abgrenzung zu den dérives der Situationistischen Internationale nicht mit einem wilden, ziellosen Umherwandern zu vergleichen (vgl. Bossert 2014; Schoch/Bossert 2017) - auch wenn die Form der Fortbewegung zu Fuß dieselbe ist. Insbesondere die schon erwähnte Langsamkeit, die die Spazierenden erfahren, fungiert als zentrales Element in Burckhardts Spaziergangswissenschaft. Dadurch ergibt sich „eine Praxis der Analyse von Räumen und Orten mit ihren Qualitäten und Funktionen als Format der Vermittlung und Darstellung“( Ritter 2019: 25 f.). Das wird in der sozialkonstruktivistischen Perspektive Burckhardts deutlich. Mit der Metapher der Perlenkette versteht Burckhardt den Spaziergang als eine Sequenz: Die während dem Spazieren aufgenommenen Eindrücke, auch metaphorisch als Perlen zu verstehen, reihen sich wie in einer Kette aneinander und führen folglich in den Köpfen der Spazierenden zu kohärenten Landschaften (Burckhardt 1995; vgl. Schoch/Bossert 2017). Das typische Landschaftsbild ist das Ergebnis einer integrativen Leistung der Aneinanderreihung von Perlen (vgl. Burckhardt 1995: 272). Manche dieser Perlen entstammen jedoch nicht ausschließlich dem Gesehenen, sondern werden hinzugedichtet, weil sie von den Spazierenden aufgrund ihrer Erfahrung und ihres Wissens, beziehungsweise durch ihren „individuellen Wahrnehmungsfilter“, als typisch für die durchwanderte Landschaft gelten
Abb. 4 Autofahrerspaziergang im Seminar, Wahrnehmung und Verkehr', Kassel 1993 (Foto: Bertram Weisshaar. @ Martin Schmitz Verlag) 
(Schoch/Bossert 2017: 323). In der Folge sehen die Spazierenden das, was sie gelernt haben. Das heißt, dass Landschaft im Kopf entsteht und nicht unbedingt durch die (alleinige) Betrachtung der Umwelt (ebd.).

In dieser Hinsicht kritisierte Burckhardt (1995: 259), „daß die Wahrnehmung gesteuert ist durch Festlegungen, die selber aus früheren Wahrnehmungen erarbeitet sind“. Für Burckhardt war es ein Anliegen, mit der Spaziergangswissenschaft „gleichzeitig mit der Wahrnehmung auch die Determiniertheit unserer Wahrnehmungsformen aufzuzeigen, so daß auch neue ungewohnte Beurteilungen altbekannter Situationen möglich werden“ (ebd.). Damit soll nicht gesagt sein, dass die bestehenden Wahrnehmungsformen als unwichtig oder falsch zu beurteilen sind. Es geht vielmehr darum, auch neue Sichtweisen auf das schon Vorgeformte, Bekannte und Vertraute zuzulassen. In diesem Spannungsverhältnis zwischen gewohnten und ungewohnten Beurteilungen der Wahrnehmung entsteht die Möglichkeit, Neues zu lernen und vertraute Wahrnehmungen kritisch zu reflektieren. Während eines Spaziergangs durch die Stadt ist die Spaziergangswissenschaft somit nicht nur ein didaktisches Mittel, sondern zugleich auch als Methode der Stadtplanungskritik zu verstehen (Schoch/Bossert 2017). Dabei ist es ihr Ziel, die „Widersprüche und Auffälligkeiten ausgewählter Stadtausschnitte oder Stadtaspekte durch eine veränderte Perspektive zutage treten zu lassen und bewusst zu machen“ (ebd.: 324). Ein Spaziergang bildet dafür einen geeigneten Rahmen.

Burckhardt sah seine neue Wissenschaft als „ein Instrument sowohl der Sichtbarmachung bisher verborgener Teile des Environments als auch ein Instrument der Kritik der konventionellen Wahrnehmung selbst“ (1995: 265). Durch die entschleunigte Fortbewegung bleibt den Spazierenden genügend Zeit, um einerseits die Umwelt zu betrachten und diese andererseits auch sinnlich auf sich zurückwirken zu lassen. Dies erlaubt die materiellen und immateriellen Artefakte genauer zu betrachten und zeitgleich kritisch zu reflektieren. Das Spazieren soll dazu animieren, festgelegte Wahrnehmungskonstruktionen des Environments zu hinterfragen und folglich auch aufzubrechen (Bürgin/Schoch 2015: 73). Was heißt das für die Spazierenden konkret? Der Spaziergang versucht das aus der Lebenserfahrung vorgefertigte Landschaftsbild im Kopf aufzulösen und neue Erfahrungen der Wahrnehmung und Reflexion zuzulassen (vgl. Schoch/Bossert 2017: 323).

Obwohl Burckhardt die Spaziergangswissenschaft als ein ,Instrument‘ betitelte, so lässt sie sich auch als Methode des „Action-Teaching“ zur Erfahrung von städtischen Kontexten im Hinblick auf die Generierung neuen Wissens über die Umwelt verstehen (Bossert 2014: 140 f.). Dieses neue Wissen zeigt sich nicht zuletzt in der kritischen Wahrnehmung der Umwelt, sei diese städtisch oder auch ländlich geprägt. Die Spaziergangswissenschaft animiert dazu, das Gesehene beispielsweise in einem Spannungsverhältnis von Mensch-Umwelt-Politik zu reflektieren (vgl. Burckhardt 1967). Dieses zunächst deskriptive Vorgehen ermöglicht in der darauf aufbauenden kritischen Reflexion in der systemischen Denkweise eine durchaus kritische wie auch innovative Methode der Wissensgenerierung.

Mit Burckhardts Vorhaben, auf dem Spazieren eine eigene Disziplin zu begründen, kritisiert er die Methode zugleich. So ist sie thematisch wenig eingegrenzt und eng mit anderen Disziplinen und ethnographischen Methoden verwandt. Zudem besteht die Gefahr, dass die promenadologischen 
Spaziergänge vermehrt einem Spektakel gleichen, insofern deren sorgfältige Vorbereitung übersehen beziehungsweise übergangen wird (Bossert 2014: 152 f.). Darüber hinaus unterliegen die Erkenntnisse aus der Spaziergangswissenschaft der subjektiven Wahrnehmung der Spazierenden. Das heißt, die Wahrnehmungen und Erkenntnisse sind abhängig von deren Vorkenntnissen, Bildern und Wertevorstellungen.

\section{Wissen, aber unkonventionell}

Burckhardt war wohl für damalige sowie heutige Hochschulverhältnisse ein eher unkonventioneller Professor. Er mied weitgehend wissenschaftliche Diskurse und Konventionen - seien diese inhaltlicher oder methodischer Natur. Anstatt sich an maßgeschneiderten, standardisierten Methoden zur Erforschung der Umwelt zu orientieren, gründete er kurzum seine eigene und erweiterte sie um den Begriff,Wissenschaft'. Burckhardt ließ sich dabei nicht in ein herkömmliches Methodenkorsett schnüren. Die Spaziergangswissenschaft selbst scheint bis heute keineswegs standardisiert zu sein. Sie ist stets von den subjektiven Erfahrungen und der Sozialisierung der Spazierenden abhängig. Womöglich ist dies auch ein Grund dafür, dass die Suche nach der Spaziergangswissenschaft, so wie sie Burckhardt lehrte, in den Handbüchern zur qualitativen und ethnographischen Forschung vergeblich bleibt. Nichtsdestotrotz sind und bleiben meiner Ansicht nach Spaziergänge ein nützliches Mittel zur Gewinnung von Eindrücken und kritischer Reflexion. Sie können helfen, physische Räume zu erkunden und erste Eindrücke eines zukünftigen oder aktuellen Forschungsgegenstandes zu generieren. Durch solche Spaziergänge wird es möglich, neue Fragen über den Forschungsgegenstand aufzuwerfen, die nicht aus der Literatur stammen und die beispielsweise in darauffolgenden qualitativen Interviews während eines Forschungsprojekts weiter vertieft werden könnten. Dieses Unterfangen hat sich auch in meiner Forschung zum Planungskonflikt im Basler Hafenareal bewährt (Bürgin 2017). Denn tatsächlich sieht die Welt vor Ort anders aus als am Schreibtisch. Ob auf einem Spaziergang durch einen städtischen Konfliktraum oder beim Mitlaufen an einem Protestmarsch der Spaziergang hilft, den Blick von Forschenden zu schärfen, dialektisch die eigene Wahrnehmung zu schulen und neue Fragen aufzuwerfen. Lucius Burckhardt hat uns gelehrt, mehr zu sehen, das Gesehene zu verstehen und dadurch das eigene Wissen zu erweitern.

\section{Endnoten}

[1] Der Text dieses Beitrags steht unter der Lizenz CC BY-SA 4.o, die Bildrechte liegen beim Verlag Martin Schmitz.

[2] Siehe dazu ausführlich die Debatte zu Lucius Burckhardt (1974), „Wer plant die Planung?“ in sub \urban 5/1-2 von 2017. 


\section{Autor_innen}

Reto Bürgin ist Sozialgeograf und forscht zu Digital Urban-Rural-Linkages, Stadtgeografie/ -soziologie und den Schriften von Lucius Burckhardt.

reto.buergin@giub.unibe.ch

\section{Literatur}

Bossert, Markus (2014): Spazieren als Wissenschaft. In: Ueli Mäder / Peter Sutter / Markus Bossert / Aline Schoch / Reto Bürgin / Simon Mugier / Hector Schmassmann (Hg.), Raum und Macht: Die Stadt zwischen Vision und Wirklichkeit. Leben und Wirken von Lucius und Annemarie Burckhardt. Zürich: Rotpunktverlag, 139-153.

Burckhardt, Lucius (1967): Bauen - ein Prozess ohne Denkmalpflichten. In: Jesko Fezer / Martin Schmitz (Hg.), Wer plant die Planung? Architektur, Politik und Mensch. Berlin: Martin Schmitz, 26-45.

Burckhardt, Lucius (1974): Wer plant die Planung? In: Jesko Fezer / Martin Schmitz (Hg.), Wer plant die Planung? Architektur, Politik und Mensch. Berlin: Martin Schmitz, 71-88.

Burckhardt, Lucius (1994): Können wir die Stadt wahrnehmen? In: Helmut Aebischer (Hg.), das ZEBRA streifen. Kassel: Infosystem Planung, Gesamthochschule Kassel, 4-5.

Burckhardt, Lucius (1995): Spaziergangswissenschaft. In: Markus Ritter / Martin Schmitz (Hg.), Warum ist Landschaft schön? Die Spaziergangswissenschaft. Berlin: Martin Schmitz, 257-300.

Burckhardt, Lucius (2017): Können wir die Stadt wahrnehmen? In: Noah Regenass / Markus Ritter / Martin Schmitz (Hg.), Landschaftstheoretische Aquarelle und Spaziergangswissenschaft. Berlin: Martin Schmitz, 349-350.

Bürgin, Reto (2017): Interessenskonflikt im Basler Hafenareal. Wie Widerständige Lucius Burckhardts Planungskritik neu interpretieren. In: sub \urban. zeitschrift für kritische stadtforschung 5/1-2, 277-286.

Bürgin, Reto (2019): Lucius Burckhardt als Vordenker eines sozio-materiellen Raumverständnisses. In: Monika Kurath / Reto Bürgin (Hg.), Planung ist unsichtbar: Stadtplanung zwischen relationaler Designtheorie und Akteur-Netzwerk-Theorie. Bielefeld: transcript, 39-66.

Bürgin, Reto / Schoch, Aline (2015): Wie Spazieren Wissen schafft: Über die Spaziergangswissenschaft nach Lucius und Annemarie Burckhardt. In: Archithese - Internationale Zeitschrift und Schriftenreihe für Architektur: Architektur und Soziologie 2/45, 72-79.

Gribat, Nina (2017): Lucius Burckhardt: Der kleinstmögliche Eingriff. In: Frank Eckardt (Hg.), Schlüsselwerke der Stadtforschung. Wiesbaden: Springer, 145-160.

Ritter, Markus (2019): Weltwahrnehmen - Weltzurichten: Die Programmatik der Spaziergangswissenschaft von Lucius Burckhardt. In: Annie Pfeifer / Reto Sorg (Hg.), „Spazieren muss ich unbedingt“: Robert Walser und die Kultur des Gehens. Paderborn: Wilhelm Fink, 17-26.

Schoch, Aline / Bossert, Markus (2017): Spazierend zur Emanzipation? Die Spaziergangswissenschaft als Instrument innovativer Stadterkundung. In: Patrick Oehler / Nadine Käser / Matthias Drilling / Jutta Guhl / Nicola Thomas (Hg.), Emanzipation, Soziale Arbeit und Stadtentwicklung. Opladen u. a.: Budrich, 321-337.

Sutter, Peter (2014): Lucius Burckhardt-Wackernagel. In: Ueli Mäder / Peter Sutter / Markus Bossert / Aline Schoch / Reto Bürgin / Simon Mugier / Hector Schmassmann (Hg.), Raum und Macht: Die Stadt zwischen Vision und Wirklichkeit. Leben und Wirken von Lucius und Annemarie Burckhardt. Zürich: Rotpunktverlag, 21-70. 
